\title{
Is there a link between pulmonary disease and bone density in older men?
}

\author{
“...changes in skeletal muscle mass and BMD in the 'healthy' aging \\ population have been demonstrated to occur in various chronic \\ inflammatory conditions, both of the lung and other sites, including chronic \\ heart failure and rheumatoid disease."
}

As part of the natural aging process, a change occurs in body composition with the loss of body mass, fat mass and fat-free mass (FFM), all of which have been associated with loss of BMD, including osteoporosis. There are gender differences in these changes that may relate to variation between the sexes in the distribution of these components of body composition. In addition, in women, the postmenopausal status has an impact. However, in both sexes, a key change that occurs with aging is the loss of skeletal muscle mass - sarcopenia - which has been linked to loss of BMD. Males with a longer period of eugonadal status and greater skeletal muscle mass are considered to be partially protected from loss of BMD. If this is generally true, what other factors could be active in the development of bone thinning in older men? In this editorial, I will develop the hypothesis that undiagnosed mild-severity pulmonary disease, in particular chronic obstructive pulmonary disease (COPD), could be a major factor in BMD decline and is deserving of further research because such patients could benefit from currently available therapeutic interventions.

\section{"As part of the natural aging process, a change occurs in body composition with the loss of body mass, fat mass and fat-free mass ... all of which have been associated with loss of BMD, including osteoporosis."}

The associated changes in skeletal muscle mass and BMD in the 'healthy' aging population have been demonstrated to occur in various chronic inflammatory conditions, both of the lung and other sites, including chronic heart failure and rheumatoid disease. In the setting of pulmonary disorders, alterations in body composition have been reported in acute lung injury requiring prolonged intensive care, cystic fibrosis and COPD, all of which have been associated with systemic inflammation [1-3]. Such changes occurring in inflammatory disease states have been interpreted as premature or accelerated biological aging. In COPD patients, aortic stiffness was greater than in control subjects and the suggested accelerated aging may occur in the central vasculature. Interestingly, the stiffest aortas were recorded in patients with osteoporosis [4]. Recently, in patients of both sexes with COPD, an undiagnosed loss of BMD was associated with a loss of FFM across the severity range of airways obstruction $[5,6]$. In a group of 81 patients ( 43 male), $72 \%$ had either osteoporosis or osteopenia at the total hip site and $65 \%$ demonstrated the same at the total lumbar site; by contrast, in 38 age-matched control subjects (19 male), $40 \%$ had either osteoporosis or osteopenia at the hip and $34 \%$ at the lumbar site. Importantly, a high level of bone thinning was observed in patients with osteoporosis or osteopenia at the total hip (63\%) and lumbar spine (56\%) sites in males compared with 82 and $76 \%$, respectively, in the females. The FFM index was the only predictor of BMD at the hip and lumbar sites from a group of variables including IL-6, smoking pack years and lung function; none of these variables were predictive in the control subjects. An indicator of the relationship between FFM and low BMD is the finding that $21 \%$ of the patients had a low FFM and a normal BMI, and, of this group, $88 \%$ had either osteoporosis or osteopenia. Using surrogate urinary biomarkers of cellular and bone connective tissue protein breakdown, 5-ribosyl uracil (pseudouridine) and $N$-telopeptides of collagen-1, Bolton et al. reported increased cellular protein breakdown in patients with COPD, which was greatest in those with a low FFM, while increased bone protein breakdown was associated with the presence of osteoporosis.

In COPD, changes in body composition including loss of BMD are likely to be related to the natural aging process and to disease-related
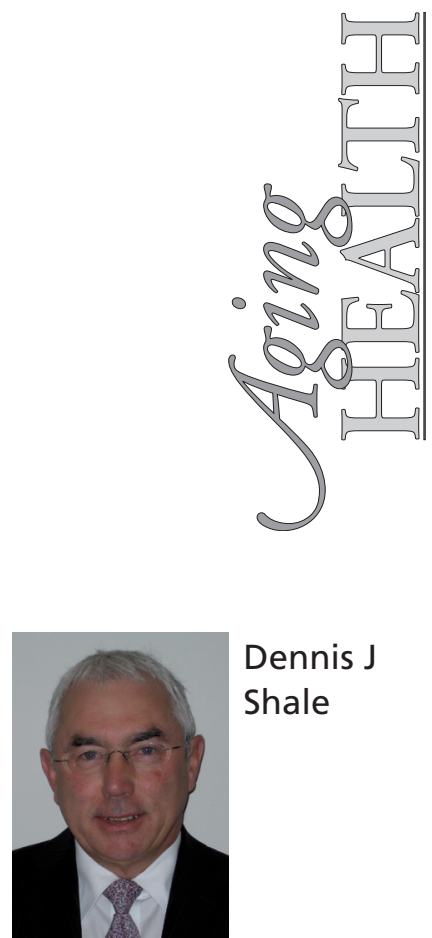

Section of Respiratory Medicine, Wales Heart Research Institute, School of Medicine, Cardiff University, University Hospital of Wales, Heath Park, Cardiff, CF14 4XN, UK Tel.: +442920716948 Fax: +442920716416 shaledj@cardiff.ac.uk

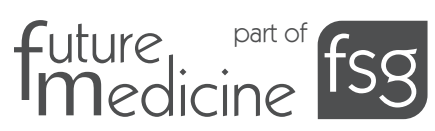


effects. Support for a disease-related component comes from a study of steroid-naive men with COPD, in which nearly $50 \%$ were demonstrated to have at least one lumbar spinal fracture. Recently, Duckers et al. reported, in a small cohort of both inhaled and oral corticosteroid-naive males compared with smokingmatched controls, evidence of either osteoporosis or osteopenia in $73 \%$ of the inhaled and oral corticosteroid-naive group compared with 40\% in control subjects [7]. Again, FFM was related to $\mathrm{BMD}$ and was a predictor of $\mathrm{BMD}$, and there was evidence of increased bone turnover based on circulating biomarkers that were inversely linked to BMD. Importantly, $80 \%$ of the patients were of mild severity and $17 \%$ would not have been diagnosed with COPD using UK criteria. The disease-specific components may include a reduction in physical activity, increased energy and oxygen costs of breathing, chronic systemic inflammation and catabolic intermediary metabolism, nutritional impairment, tobacco smoking and occupational exposures. Some of these factors lead to extra energy costs, with a resting energy expenditure increase of approximately $20 \%$ in COPD patients. The overall nutritional status is determined by the balance between the energy costs and the energy intake required to meet those costs. Nutrient intake has been reported to meet the requirements recommended for healthy subjects, but the excess requirements of energy in the disease state mean that these costs may not be fully met by nutrient intake, leading to a negative energy balance and a call on endogenous energy stores to meet the deficit. In this area, COPD and other chronic inflammatory states may utilize proteinrich tissues in order to meet energy demands, a metabolic response that is the reverse of the conservation response seen in simple starvation. Thus, inappropriate protein catabolism with reduced physical activity may underlie the loss of skeletal muscle mass and function with parallel loss of BMD.

Could there be a large male population with undiagnosed pulmonary disease? The likely answer is yes, because in healthy populations, epidemiological data links reduced lung function and airways obstruction with cardiovascular morbidity and mortality, which are also linked very closely to the presence of osteoporosis. In addition, COPD is not well recognized in its mildest forms and is likely to go undiagnosed for long periods. It has been estimated that in the UK, there are 900,000 patients with diagnosed COPD out of a potential pool of 3.7 million, a situation that is not peculiar to the UK. These 'missing millions', as described by the British Lung Foundation (London, UK) [8], represent a significant proportion of older patients who may demonstrate relatively modest respiratory symptoms and signs, but on screening, a high proportion could be found to have obstructive airways disease related to industrial exposure or a smoking habit and these individuals are likely to have milder-severity COPD. However, even mildseverity COPD with a normal BMI and therefore body weight, and moderately well-preserved lung function, may well have an undiagnosed loss of BMD. Thus, it is possible that a proportion of men developing loss of BMD and frank osteoporosis develop their problem as a consequence of 'hidden' or undiagnosed lung disease.

"It has been estimated that in the UK, there are 900,000 patients with diagnosed COPD out of a potential pool of 3.7 million, a situation ... not peculiar to the UK."

Such individuals can be detected by focused screening using spirometry, anthropometric measures and validated questionnaires, so the hypothesis that a high proportion of older males with a low or reduced BMD have undiagnosed pulmonary disease could be tested in a population study [9]. The importance of this possible relationship is that there are interventions, such as muscle reconditioning through exercise programs, that may help to maintain peripheral skeletal muscle mass and offer some bone protection, particularly if they are applied earlier than we currently recommend for pulmonary rehabilitation. Another intervention includes the direct treatment of low vitamin $\mathrm{D}$ status and reduced BMD with agents such as bisphosphonates.

\section{"...it is possible that a proportion of men developing loss of BMD and frank osteoporosis develop their problem as a consequence of ‘hidden' or undiagnosed lung disease."}

There are several sources of evidence that taken individually seem circumstantial; however, they support the hypothesis put forward here, and, taken together, they constitute a plausible explanation of one factor underlying bone density loss in older males. 


\section{Financial \& competing interests disclosure}

The author has no relevant affliations or financial involvement with any organization or entity with a financial interest in or financial conflict with the subject matter or materials discussed in the manuscript. This includes employment, consultancies, honoraria, stock ownership or options, expert testimony, grants or patents received or pending, or royalties.

No writing assistance was utilized in the production of this manuscript.

\section{Bibliography}

1. Ionescu AA, Nixon LS, Evans WD et al:: Bone density, body composition and inflammatory status in cystic fibrosis. Am. J. Resp. Crit. Care Med. 162, 789-794 (2000).

2. Eid AA, Ionescu AA, Nixon LS et al.: The inflammatory response and body composition in chronic obstructive pulmonary disease. Am. J. Resp. Crit. Care Med. 164, 1414-1418 (2001).

3. Ionescu AA, Nixon LS, Luzio $S$ et al.: Pumonary function, body composition and protein catabolism in adults with cystic fibrosis. Am. J. Resp. Crit. Care Med. 165, 495-500 (2002).

4. Sabit R, Bolton CE, Edwards PH et al: Arterial stiffness and osteoporosis in chronic obstructive pulmonary disease. Am. J. Resp. Crit. Care Med. 175, 1259-1265 (2007).
5. Bolton CE, Ionescu AA, Shiels KM et al.: Associated loss of fat free mass and bone mineral density in chronic obstructive pulmonary disease. Am. J. Resp. Crit. Care Med. 170, 1286-1293 (2004).

6. Kjensli A, Mowinckel P, Ryg MS, Falch JAL Low bone mineral density is related to severity of chronic obstructive pulmonary disease. Bone 40, 493-497 (2007).

7. Duckers JM, Bolton CE, Evans BAJ, Evans WD, Stone MD, Shale DJ:

Osteoporosis and loss of bone mineral density in corticosteroid naïve men with chronic obstructive pulmonary disease. Thorax 63(Suppl. VII) A48 (2008).

8. Jarrold I, Eiser N, Lethbridge T: British Lung Foundation's search for the missing millions of COPD patients. Thorax 64, A154-A159, 185 (2009).
9. Bolton $\mathrm{CE}$, Cannings-John R, Edwards $\mathrm{PH}$ et al:: What community measurements can be used to predict bone disease in patients with COPD. Resp. Med. 102, 651-659 (2008).

\section{Affiliation}

- Dennis J Shale

Section of Respiratory Medicine, Wales Heart Research Institute, School of Medicine, Cardiff University, University Hospital of Wales, Heath Park, Cardiff, CF14 4XN, UK Tel.: +442920716948 Fax: +442920716416 shaledj@cardiff.ac.uk 